\title{
铁系胶体催化剂各组分相互作用的研究 *
}

\author{
夏树伟夏少武 ${ }^{1}$ 宋昭峥 ${ }^{1}$ \\ (青岛海洋大学化学化工学院, 青岛 266003; 1 青岛化工学院应用化学系, 青岛 266042)
}

关键词：胶体催化剂, 电导率, 紫外 - 可见光谱

已知催化剂 $\mathrm{FeCl}_{3}-\mathrm{Al}(i-\mathrm{Bu})_{3}-\mathrm{Phen}$ (邻菲罗啉) 在加氢汽油介质中对丁二烯聚合有较高的活 性 ${ }^{[1-2]}$, 作者曾研究了 $\mathrm{FeCl}_{3}-\mathrm{Al}(i \text { - } \mathrm{Bu})_{3}$-Phen 体系催 化剂的相态,证明该体系为胶体催化剂 ${ }^{[3]}$.

Bushick $^{[4]}$ 较早地使用电导率研究烷基铝 - 氯化 钛体系离子特性与聚合速率的关系. 至今尚未见从 胶体角度研究本体系各组分之间相互作用的报导。

本文是把电导率作为胶体的电学性质之一进行 研究的。主要研究了 $\mathrm{FeCl}_{3}-\mathrm{Al}(i-\mathrm{Bu})_{3}$-Phen 胶体催 化剂体系单、多组分溶液的电导率与浓度的关系, 结 合相应的紫外 - 可见光谱分析结果, 得出 $\mathrm{Al}(i-\mathrm{Bu})_{3}$ 不仅是还原剂, 而且还能形成双电层具有稳定催化 剂胶粒作用的结论.

\section{1 实验部分}

原料: 无水三氯化铁简记为 $(\mathrm{Fe})$,三异丁基铝简 记为 $(\mathrm{Al})$, 邻菲罗啉简记为 (Phen), 丁二烯加氢汽油 溶液简记为 $(\mathrm{Bd})$, 具体规格和性能, 可参见文献 [3].

电导率与紫外 - 可见光谱的测定参见文献 [5].

\section{2 结果与讨论}

\section{1 单组分的电导率及紫外 - 可见光谱}

测得加氢汽油在 $25^{\circ} \mathrm{C}$ 时电导率为 $1.0 \times 10^{-8} \mathrm{~S}$. $\mathrm{m}^{-1}$, 比相同条件下纯水电导率 $6.63 \times 10^{-6} \mathrm{~S} \cdot \mathrm{m}^{-1}$ 低得多. $(\mathrm{Fe})$ 和 $(\mathrm{Phen})$ 的电导率很小, 接近于 0 , 其 值几乎不随浓度的变化而变化, 这表明 $(\mathrm{Fe})$ 和 (Phen) 几乎不发生电离. ( $\mathrm{Al}$ ) 的电导率值较高 (见 图 1(I)), 且随浓度增加而增大。浓度低时曲线上 升较快, 浓度较高时上升较缓慢。
三价铝化合物是缺电子化合物, Bushick ${ }^{[4]}$ 根据 电导率测定提出 $\mathrm{Al}(i-\mathrm{Bu})_{3}$ 常常缔合为二聚或多 聚, 并进一步解离成离子对, 如式 (1) 所示 (式中 $\mathrm{R}$ 代表 $i$ - $\mathrm{Bu}$ 基).

$2 \mathrm{AlR}_{3} \leftrightarrows\left(\mathrm{AlR}_{4}\right)^{-}\left(\mathrm{AlR}_{2}\right)^{+} \leftrightarrows\left(\mathrm{AlR}_{4}\right)^{-}+\left(\mathrm{AlR}_{2}\right)^{+}$

由于解离平衡的存在, 图 1 中 $(\mathrm{Al})$ 的电导率随 着浓度的进一步增大, 上升逐渐缓慢。

单组分 $(\mathrm{Fe})$ 为黄色, 其紫外 - 可见光谱在 $200-800 \mathrm{~nm}$ 范围内有三个吸收峰, 分别是 228. 8 、 $271.7 、 360.8 \mathrm{~nm}$, 归属为 $\mathrm{Cl} \rightarrow \mathrm{Fe}(\mathrm{III})$ 荷移跃迁, 是 $\mathrm{FeCl}_{3}$ 的特征吸收峰; (Phen) 在 $302.0 \mathrm{~nm}$ 和 323.0

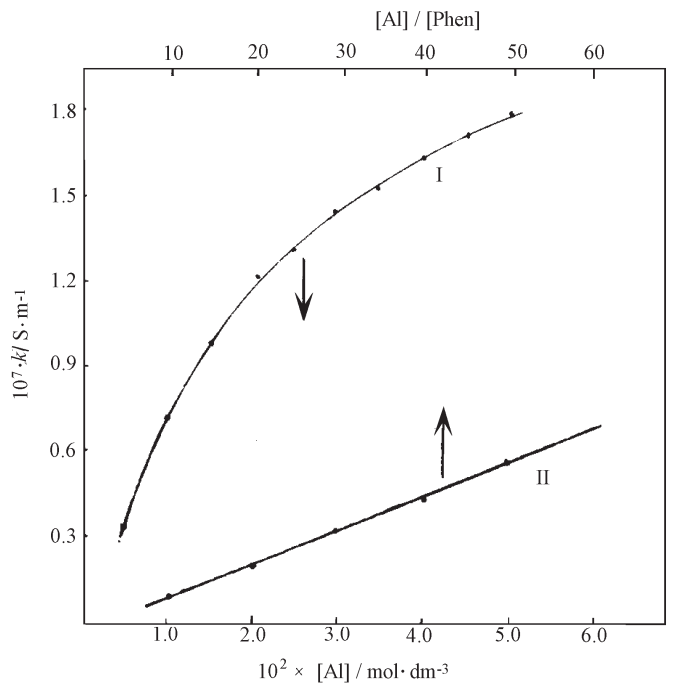

图 1 各组分电导率与浓度的关系 $\left(25^{\circ} \mathrm{C}\right)$

Fig. 1 The relationship of the electric conductivity of various components with concentration

(1) the electric conductivity of single-component $(\mathrm{Al})$,

(2) the electric conductivity of bi-component (Al)-(Phen) 
$\mathrm{nm}$ 处有两个吸收峰, 归属为 (Phen) 中 $\mathrm{C}=\mathrm{C}$ 和 $\mathrm{C}=$ $\mathrm{N}$ 键的 $\pi \rightarrow \pi^{*}$ 跃迁.

\section{2 双组分体系的电导率及光谱}

\section{$2.2 .1(\mathrm{Fe})-(\mathrm{Phen})$ 的电导率及光谱}

当一定量的 $(\mathrm{Phen})$ 加人 $(\mathrm{Fe})$ 后, 溶液呈淡黄色. (Fe)-(Phen) 双组分的 UV-Vis 光谱如图 2 所示, 当 按 $[\mathrm{Phen}] /[\mathrm{Fe}]=1$ 混合时, $(\mathrm{Fe})$ 在波长为 360.8 $\mathrm{nm}$ 处的特征峰消失, 在波长为 $263.8 \mathrm{~nm}$ 处出现一 个新的吸收峰, 在 $316.0 \mathrm{~nm}$ 处出现一个肩峰. 随 $[\mathrm{Phen}] /[\mathrm{Fe}]$ 摩尔比值的增大, 吸收峰逐渐右移, 吸 收峰的高度也逐渐升高. 这是因为 (Phen) 有较强 的配位能力, 能与 $\mathrm{FeCl}_{3}$ 生成 $\mathrm{Fe}^{3+} \leftarrow \mathrm{Phen}$ 配合物 . 随着 (Phen) 量的增加, 生成配合物浓度增加, 因此峰 高增加. 文献 [3] 已证明 $(\mathrm{Fe})+(\mathrm{Phen})$ 在加氢汽油 介质中为直径 $60 \mathrm{~nm}$ 左右的胶粒, 通常认为 (Phen) 与胶粒表面缺陷部位的 $\mathrm{Fe}^{3+}$ 配位的可能性大, 配位 的结果降低表面能. 随表面配合物浓度的增加, 表 面能继续降低, 因此产生红移.

通过以上分析可看出, 波长分别在 $263.8 \mathrm{~nm}$ 和 $316.0 \mathrm{~nm}$ 处的两个峰是与颗粒表面的 $\mathrm{Fe}^{3+} \leftarrow \mathrm{Phen}$ 配合物有关的吸收峰.

文献 [6] 给出了 (Phen) 与 $(\mathrm{Fe})$ 生成的配合物为 $\mathrm{Fe}(\mathrm{Phen})_{n} \mathrm{Cl}_{3}$, 故认为 $\mathrm{FeCl}_{3}$ 颗粒的表面反应为

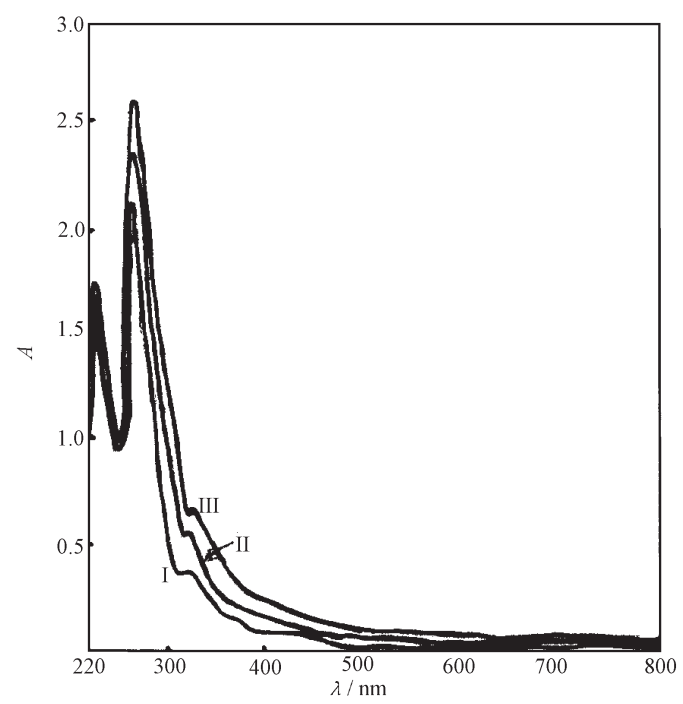

图 2 (Fe)-(Phen) 的 UV-Vis 光谱

Fig. 2 The UV-Vis spectrum of bi-component (Fe)-(Phen )

Conditions: $\left.[\mathrm{Fe}]=5.55 \times 10^{-5} \mathrm{~mol} \cdot \mathrm{dm}^{-3}, \mathrm{I}\right)[\mathrm{Phen}] /$ $[\mathrm{Fe}]=1$, II $)[\mathrm{Phen}] /[\mathrm{Fe}]=2$, III $)[\mathrm{Phen}] /[\mathrm{Fe}]=3,25^{\circ} \mathrm{C}$

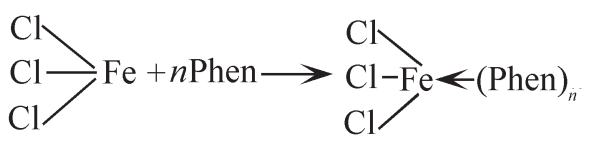

式中 $n$ 值随 $[\mathrm{Phen}] /[\mathrm{Fe}]$ 比值不同, 可能取 1 或 3 .

在 $[\mathrm{Phen}] /[\mathrm{Fe}]$ 为 $0.5 \sim 3.0$ 浓度范围内, $(\mathrm{Phen})+(\mathrm{Fe})$ 的电导率随 $(\mathrm{Phen})$ 浓度的增加几乎不 变, 这说明 $\mathrm{Fe}(\mathrm{Phen}){ }_{n} \mathrm{Cl}_{3}$ 不发生解离.

\subsection{2 (Al)-(Phen) 双组分的电导率与光谱}

在一定浓度 $(\mathrm{Phen})$ 中加人 $(\mathrm{Al})$, 溶液呈粉红色. 电导率变化如图 1 中 ( II) 所示. 由图可见电导率随 [Al] / [Phen] 摩尔比值的增大呈线性增加. 此时 (Al)的浓度较低, 相当于图 1 中曲线 ( I) 的起始部 分. 比较图中 (I) 与 (II) 可知, 双组分的电导率小于 同浓度单组分 $(\mathrm{Al})$ 的电导率, 这预示出 (Phen) 与 (Al) 之间发生作用. (Al) - ( (Phen) 双组分 UV-Vis 光谱与图 2 相似, 随着 [Al]/[Phen] 比值增加, 位于 278. $1 \mathrm{~nm}$ 的吸收峰高度增加, 认为吸收峰是配体 $\mathrm{Phen} \rightarrow \mathrm{Al}$ (III) 荷移所至. 结合电导率与紫外 - 可见 光谱实验结果推断 $(\mathrm{Al})$ 与 $(\mathrm{Phen})$ 发生配位反应:

$$
\mathrm{Phen}+\mathrm{AlR}_{3} \rightarrow \mathrm{Al}(\text { Phen }) \mathrm{R}_{3}
$$

Fisenka ${ }^{[7]}$ 由 NMR、EPR 研究也发现在 Phen 与 $\mathrm{AlR}_{3}$ 之间发生反应.

\section{$2.2 .3(\mathrm{Fe})-(\mathrm{Al})$ 双组分的电导率与光谱}

在一定量的 $(\mathrm{Fe})$ 中加人 $(\mathrm{Al})$ 溶液立刻变成黑 色, 混合后的电导率随 $(\mathrm{Al})$ 浓度的变化如图 3(I) 所 示. 摩尔比 $[\mathrm{Al}] /[\mathrm{Fe}]=10.0 \sim 60.0$ 范围内, 电导 率逐渐增加, 当 $[\mathrm{Al}] /[\mathrm{Fe}]=66.0$ 时, 降至极小值, 当 $[\mathrm{Al}] /[\mathrm{Fe}]>70.0$ 时电导率逐渐升高.

在 $[\mathrm{Al}] /[\mathrm{Fe}]=66.0$ 时为较佳配比,生成的催 化剂颗粒较小, 粒径约 $30 \mathrm{~nm}$ 左右, 属胶体范围. $(\mathrm{Fe})-(\mathrm{Al})$ 紫外 - 可见吸收光谱也证明了这一点 (图 4). 在 $350 \mathrm{~nm}$ 处出现吸收峰, 且随 $[\mathrm{Al}] /[\mathrm{Fe}]$ 摩尔 比增加, 吸收峰逐渐升高, 吸收峰位置发生紫移. 这 是因为 $\mathrm{FeCl}_{3}$ 起初形成较大的颗粒, 随 $(\mathrm{Al})$ 的增加 反应程度增强, 胶体颗粒逐渐减小, 比表面增加, 表 面能增高,因而产生紫移。

已知 $\mathrm{AlR}_{3}$ 是很强的还原剂, 它可以把胶粒表面 的 $\mathrm{Fe}(\mathrm{III})$ 还原成低价态 $(\mathrm{Fe}(\mathrm{II}), \mathrm{Fe}(0))$, 文献 [8] 给 出本催化体系活性位是二价的。认为发生如下还原 反应:

$\mathrm{FeCl}_{3}+\left[\mathrm{AlR}_{4}\right]^{-}\left[\mathrm{AlR}_{2}\right]^{+} \rightarrow \mathrm{FeCl}_{2}\left[\mathrm{AlR}_{4}\right]+\mathrm{AlR}_{2} \mathrm{Cl}$ (4) 其中有些 $\mathrm{FeCl}_{2}\left[\mathrm{AlR}_{4}\right]$ 解离出自由基 $\mathrm{R} \cdot, \mathrm{Fe}(\mathrm{III})$ 被 


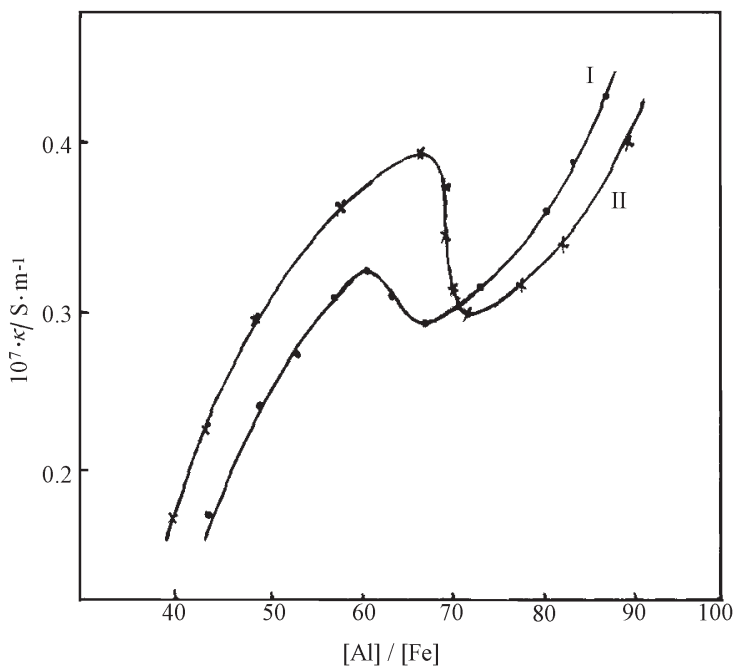

图 3 几组分混合后电导率与浓度的关系

Fig. 3 The relationship of the electric conductivity of the mixtures with concentration

Conditions: $\mathrm{I})(\mathrm{Al})+(\mathrm{Fe}), \mathrm{II})(\mathrm{Al})+(\mathrm{Fe})+($ Phen $)$, $[$ Phen $]=9.2 \times 10^{-4} \mathrm{~mol} \cdot \mathrm{dm}^{-3},[\mathrm{Fe}]=5.55 \times 10^{-5}$ $\mathrm{mol} \cdot \mathrm{dm}^{-3}, 25^{\circ} \mathrm{C}$

还原成 $\mathrm{Fe}$ (II)：

$\mathrm{FeCl}_{2}\left[\mathrm{AlR}_{4}\right] \rightarrow \mathrm{FeCl}_{2} \mathrm{AlR}_{3}+\mathrm{R} \cdot$

因此认为反应 (4)、(5) 是生成活性位的关键反 应, 由于此反应只发生在胶粒表面,即胶粒表面有一 定量的 $\mathrm{Fe}(\mathrm{II})$. 但胶粒的主体仍然是 $\mathrm{FeCl}_{3}$, 故把胶 核写成 $\left(\mathrm{FeCl}_{3}\right)_{m}$. 根据 Fajans 规则, 能与晶体组成离 子形成不溶物的离子将优先吸附。根据式(1), 胶核 将吸附过量的 $(\mathrm{Al})$ 中的 $\left[\mathrm{AlR}_{4}\right]$-离子, 使胶粒表面带 负电, 即胶粒带电. 胶粒在电场作用下移动产生相 应电导. 当 $[\mathrm{Al}] /[\mathrm{Fe}]=60$ 时, 胶粒表面电荷达到 最大值. 当 $[\mathrm{Al}] /[\mathrm{Fe}]$ 比值再增加, 开始吸附相反 离子 $\left[\mathrm{AlR}_{2}\right]+$ 使颗粒表面电荷下降, 当 $[\mathrm{Al}] /[\mathrm{Fe}]=$ 66.0 表面电荷降到极小值, 表面双电层形成了, 即 形成了稳定胶粒。因此催化剂胶团具有如下结构:

$\left[\left(\mathrm{FeCl}_{3}\right)_{m} n\left(\mathrm{AlR}_{4}^{-}\right)(n-x) \mathrm{AlR}_{2}^{+}\right]^{x-} x \mathrm{AlR}_{2}^{+}$

双电层的形成使胶粒稳定,不易发生聚结,所以 在 $[\mathrm{Al}] /[\mathrm{Fe}]=66.0$ 时制得的催化剂颗粒较小且大 小均匀; 当 $[\mathrm{Al}] /[\mathrm{Fe}]>66.0$ 时, 必定有更多的相反 离子进人 Stern 层, 使胶粒稳定性下降, 颗粒聚结变 大。由于 $(\mathrm{Al})$ 的量增多, 汽油溶液中“自由”离子增 加, 因此电导率上升。

\section{3 三组分体系电导率与光谱}

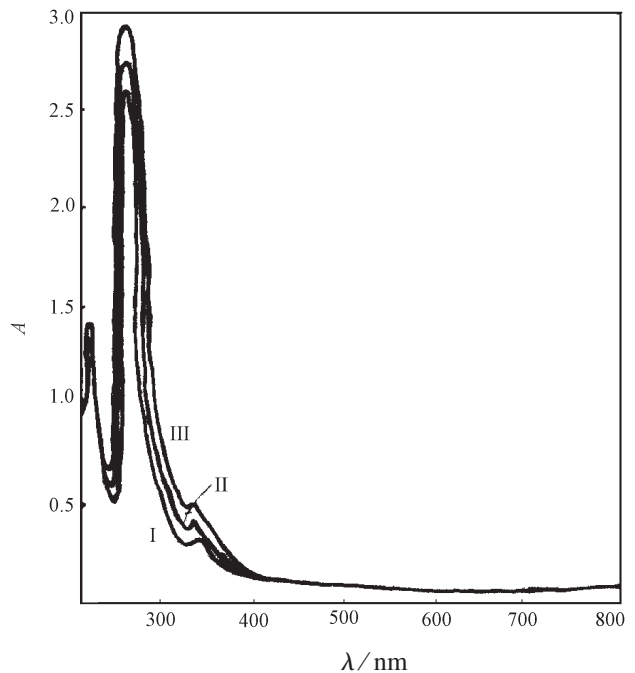

图 4 (Fe)-(Al) 双组分 UV-Vis 光谱

Fig. 4 The UV-Vis spectrum of bi-component (Fe)-(Al)

Conditions: $\mathrm{I})[\mathrm{Al}] /[\mathrm{Fe}]=50, \mathrm{II})[\mathrm{Al}] /[\mathrm{Fe}]=60$, III $[\mathrm{Al}] /[\mathrm{Fe}]=66$, [Phen $]=9.2 \times 10^{-4} \mathrm{~mol} \cdot \mathrm{dm}^{-3}$, $[\mathrm{Fe}]=5.55 \times 10^{-5} \mathrm{~mol} \cdot \mathrm{dm}^{-3}, 25^{\circ} \mathrm{C}$

三组分混合,在 $[\mathrm{Phen}] /[\mathrm{Fe}]=1.67$ 时,改变 $[\mathrm{Al}] /[\mathrm{Fe}]$, 电导率变化如图 3(II) 所示. 比较图中 (I) 与 (II), 可以看出两曲线形状相似, 只是在较佳配 比 $[\mathrm{Al}] /[\mathrm{Fe}]=66.0$ 处, 双组分的电导率出现极小 值, 而三组分的电导率出现极大值, 三组分的极小值 出现在 $[\mathrm{Al}] /[\mathrm{Fe}]=70.0$ 处. 曲线形状相似说明在 三组分中仍以 $(\mathrm{Al})$ 与 $(\mathrm{Fe})$ 的反应为主生成胶粒。三 组分的电导率极小值出现在较高 $[\mathrm{Al}] /[\mathrm{Fe}]$ 比值 处, 因为 $(\mathrm{Al})$ 与 $(\mathrm{Phen})$ 发生反应 (见反应 $(3)$ ), 需消 耗一部分 $(\mathrm{Al})$, 所以比值增加.

三组分混合后其紫外 - 可见光谱如图 5 所示, 由图看出在 $512 \mathrm{~nm}$ 处出现新的吸收带, 它是 $\mathrm{Fe}(\mathrm{II})$ 与 Phen 作用形成稳定配合物的特征谱带。这一谱 带的出现说明了 (Phen) 在形成活性位过程中的作 用. 已知邻菲罗啉同 $\mathrm{Fe}$ ( III ) 、 Fe ( II ) 都能发生配 合作用, 但对 $\mathrm{Fe}$ (II) 则有更大的稳定作用. 因为 $\mathrm{Fe}(\mathrm{Phen})_{3}^{3+}$ 的稳定常数为 $10^{15}$, 而 $\mathrm{Fe}(\mathrm{Phen})_{3}^{2+}$ 的 稳定常数为 $10^{21}{ }^{[6]}$. 这就是说当 $\mathrm{AlR}_{3}$ 还原 $\mathrm{FeCl}_{3}$ 时, Phen 的配位作用使铁离子稳定在 Fe(II) 状态, 防止进一步被还原成低价态 $[\mathrm{Fe}(\mathrm{I}), \mathrm{Fe}(0)]$ 。增加 了活性位的浓度,因此催化活性高。

正是由于 (Phen) 有稳定 $\mathrm{Fe}$ (II), 防止其进一步 


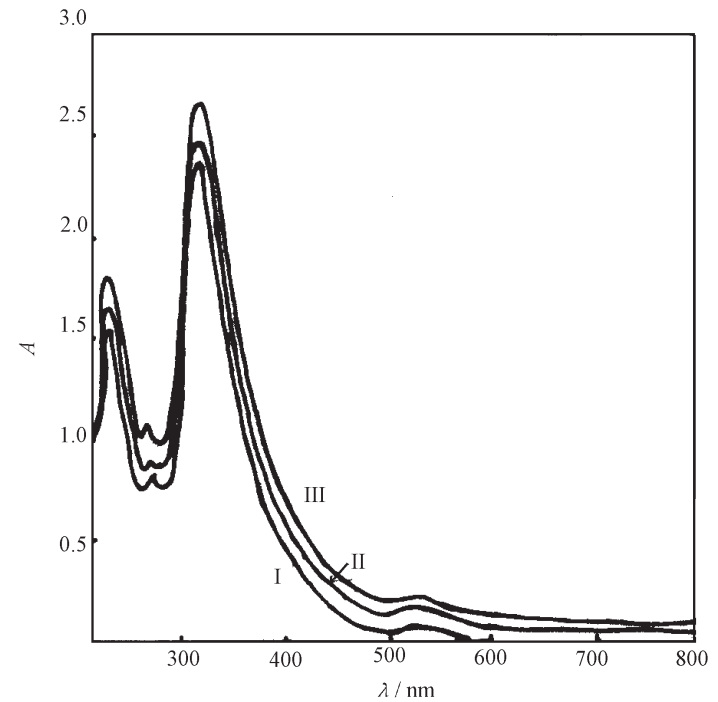

图 5 三组分的 UV-Vis 光谱

Fig. 5 The UV-Vis spectrum of tri-component system Conditions: I) $[\mathrm{Al}] /[\mathrm{Fe}]=50$, II) $[\mathrm{Al}] /[\mathrm{Fe}]=66$, III) $[\mathrm{Al}] /[\mathrm{Fe}]=70,[\mathrm{Phen}]=9.2 \times 10^{-4} \mathrm{~mol} \cdot \mathrm{dm}^{-3}$, $[\mathrm{Fe}]=5.55 \times 10^{-5} \mathrm{~mol} \cdot \mathrm{dm}^{-3}, 25^{\circ} \mathrm{C}$

被还原成低价态 $[\mathrm{Fe}(\mathrm{I}), \mathrm{Fe}(0)]$ 的作用, 在 [Al]/ $[\mathrm{Fe}]<70.0$ 时三组分的电导率大于双组分的. 当 $[\mathrm{Al}] /[\mathrm{Fe}]>70.0$ 时, 三组分电导率低于双组分的,
由于在这种条件下生成的胶粒较大 ${ }^{[3]}$, 在电场作用 下移动速率慢,因此电导率低。

\section{References}

1 Liu GZ, Wang FJ, Polymer Communications, 1984, 5: 389

[刘国智, 王风江, 高分子通讯 (Guofenzi Tongxun), 1984, 5: 389]

2 Liu GZ, Wang FJ. Polymer Communications, 1985, 4: 252 [刘国智, 王风江, 高分子通讯 (Guofenzi Tongxun), 1985, 4: $252]$

3 Xia SW, Ge XP, Li K. Chinese J. Catalysis, 1997, 18(5): 406 [夏少武, 葛小萍, 李 凯, 催化学报 (Cuihua Хиebao), 1997, 18 (5): 406 ]

4 Bushick R D, Steatns R S. J. Polymen Sci. Part A-1, 1966, 4: 215

5 Xia SW, Wei QL, Li N, et al. Acta Chimica Sinica, 1998, 56: 1159 [夏少武, 魏庆莉, 李 宁等, 化学学报 (Huaxue Xuebao), 1998, 56: 1159]

6 Xu ZG. Morden Complex Chemistry. Bejing: Chemical Industry Press, 1987, 187 [徐志固. 现代配位化学, 北京: 化学工业出 版社, 1987, 187]

7 Fisenko A Y, Grindin V A, Ershor B A, et al. Zh. Obshch. Khim., 1983, $53(2): 483$

8 Wang FJ, Zhan RY, Liu GZ, et al. Chinese J. Appl. Chem., 1989, 4 (4): 1 [王风江, 詹瑞云, 刘国智等, 应用化学 (Yingyong Hиахие), 1989, 4(4):1]

\section{Studies on the Interaction of the Various Components in Colloidal Catalysts of Iron System*}

Xia Shu-Wei Xia Shao-Wu $\quad$ Song Zhao-Zheng ${ }^{1}$

( College of Chemistry and Chemical Engineering, Ocean University of Qingdao, Qingdao 266003;

${ }^{1}$ Department of Applied Chemistry, Qingdao Chemical and Technology, Qingdao 266042)

Abstract The relationship of the electric conductivity of single and multi-component of colloidal catalyst $\mathrm{FeCl}_{3}-\mathrm{Al}(i \text {-Bu })_{3}$-Phen with their concentration was studied in hydrogenated gasoline solvent at $25^{\circ} \mathrm{C}$, meanwhile, the UV-Vis spectra of the corresponding component of the catalyst were detected. The experimental results showed that $\mathrm{Al}(i-\mathrm{Bu})_{3}$ existed in associated state and dissociated into ion couple. The reaction between $\mathrm{Al}(i \text { - } \mathrm{Bu})_{3}$ and $\mathrm{FeCl}_{3}$ was the main reaction to form colloidal core. The $\mathrm{Fe}$ (III) on the surface of the colloidal particles was reduced to lower valence by $\mathrm{Al}(i-\mathrm{Bu})_{3}$. The stable complex formed by the reaction between Phen and $\mathrm{Fe}$ (II) could prevent $\mathrm{Fe}$ (II) from being reduced to lower valence. The colloidal particles were comparatively stable by means of the electric double layer yielded by a proper excess of $\mathrm{Al}(i-\mathrm{Bu})_{3}$. The stable colloidal particles could be formed in the optimal ratio of the catalyst components.

Keywords: Colloidal catalyst, Electric conductivity, UV-Vis spectrum 